\title{
REDES DE INFORMAÇÃO: O PONTO DE CONTATO DOS SERVIÇOS E UNIDADES DE INFORMAÇÃO NO BRASIL
}

\author{
Maria Inês Tomaél
}

\section{Resumo}

Os serviços e as unidades de informação, organizados em rede, exercem uma função essencial nos processos da gestão da informação, desde a aquisição, organização, disseminação até a obtenção da informação pelo usuário final. Com o objetivo de levantar a participação dos serviços e unidades de informação em redes, no Brasil, procedemos a um levantamento, em que consultamos, especialmente, os sites das principais bibliotecas universitárias que resultou em um mapeamento das redes presentes no Brasil. Identificamos a participação brasileira em 41 redes, que foram categorizadas de acordo com sua função. A categoria que detém o maior número de redes é a de informação especializada, porém encontramos, também, redes de compatibilização da informação, redes de serviços de informação, redes de processamento de informação e redes de informação digital. A participação em rede aumenta os ativos institucionais e informacionais e na sociedade contemporânea são, cada vez mais, a base para o fortalecimento de ações.

\section{Palavras-Chave}

Redes de Informação, Serviços Cooperativos, Serviços e Unidades de Informação.

\section{INTRODUÇÃO}

Temos acesso à informação a todo o momento; mesmo sem esforço nosso a informação chega até nós por meio dos relacionamentos que mantemos ou pela mídia. A informação e o conhecimento exercem um papel que tem grande influência nas atividades econômicas, sociais e culturais, e aliados às tecnologias da in- formação concretizam a Sociedade da Informação, na qual as redes são recursos estratégicos para o desenvolvimento científico e tecnológico.

As alianças se tornam cada vez mais indispensáveis para o desenvolvimento de práticas comuns que vão facilitar e redimensionar o contexto informacional em 
que estamos inseridos. Enfim, a participação em redes, mais que uma necessidade, é hoje imprescindível para podermos desenvolver serviços e produtos em sintonia com o ambiente informacional que nos cerca e cresce a cada dia.

O relacionamento e a cooperação são inerentes ao ser humano; em todas as atividades e áreas do conhecimento, a formação de redes está fortalecendo as partes envolvidas, reduzindo os custos das operações e diminuindo o tempo gasto em cada fase do processo.

A abordagem da literatura nacional sobre a participação dos serviços e unidades de informação em redes, ultimamente, tem sido dispersa e incompleta, dificultando a visão geral. Por isso sentimos necessidade de proceder a um levantamento para reunir informações a respeito das redes de informação. Assim com o objetivo de conhecer a participação dos serviços e unidades de informação, no Brasil, em redes, realizamos um levantamento, visando mapear essas redes e identificá-las.

Os procedimentos para o levantamento da inserção dos serviços e unidades de informação em redes sucederam conforme as seguintes etapas: inicialmente consultamos o site de 35 bibliotecas de universidades federais ${ }^{1}$ e outros cinco sites

\footnotetext{
${ }^{1}$ UFAC, UFAL, UFBA, UFC, UFCG, UFES, UFF, UFG, UFJF, UFLA, UFMA, UFMG, UFMS, UFMT, UFOP, UFPA, UFPB, UFPE, UFPEL, UFPI, UFPR,
}

de bibliotecas universitárias ${ }^{2}$, de renome nacional; após, fizemos uma pesquisa no site de busca Google ${ }^{3}$ analisando as 50 primeiras indicações, para quatro pesquisas distintas ${ }^{4}$, a saber: rede informação; redes informação; "redes de informação"; "rede de informação", pesquisando apenas páginas do Brasil.

O levantamento no site das bibliotecas universitárias e no Google foi feito nos meses de maio e junho de 2004. Todas as redes identificadas foram cadastradas e as indicações de outras redes, a partir das estabelecidas inicialmente, também foram pesquisadas. Nos casos em que houve dúvida quanto às informações sobre as redes, consultamos, por e-mail, a pessoa mais indicada no âmbito da rede em questão, para responder a dúvidas específicas.

Com esse levantamento conseguimos identificar a participação brasileira em 41 redes que, de alguma forma, envolvem serviços e unidades de informação. É importante destacar que todas as redes descritas neste trabalho foram consideradas como tais por alguma das organizações pesquisadas.

\footnotetext{
UFRA, UFRGS, UFRJ, UFRN, UFPE, UFRR, UFRRJ, UFS, UFSC, UFScar, UFSJ, UFSM, UFU, UFV.

${ }^{2}$ USP, UNESP, UNICAMP, UNB, UEL.

${ }^{3}$ http://www.google.com.br

${ }^{4}$ Analisamos 200 indicações do Google, as 50 primeiras de cada pesquisa, tendo sido grande a repetição de sites. A maioria dos temas recuperados tratava de redes de computadores.
} 
Apresentamos, inicialmente, alguns conceitos que embasam as redes de informação e em seguida as categorizamos com respaldo na literatura e nas redes identificadas por este trabalho. E finalmente, relacionamos as redes que têm a participação brasileira, identificando, principalmente, suas funções básicas e seus serviços e produtos.

\section{REDES DE INFORMAÇÃO}

Redes de informação reúnem pessoas e organizações para o intercâmbio de informações, ao mesmo tempo em que contribuem para a organização de produtos e a operacionalização de serviços que sem a participação mútua, não seriam possíveis.

O tema rede está sendo abordado pela literatura em todas as suas facetas. A Internet é uma dessas redes que está sendo ponto focal de estudos e inserção na vida cotidiana das pessoas, há quase uma década. No âmbito empresarial as redes de organizações, que buscam inovação, competências e ampliação de mercado, também têm seu espaço. Mas o momento parece ser das redes sociais - redes de relacionamento - as quais, apesar de serem estudadas desde a década de 1970, agora estão sendo consideradas como recurso estratégico organizacional.

Aproveitando recursos provenientes dessas redes, as redes de informação vin- culadas a serviços e unidades de informação, têm um papel determinante em todo o processo da gestão da informação, desde a aquisição, organização, disseminação, recuperação até a obtenção da informação pelo usuário final.

Muitos são os termos empregados para conceituar e denominar as redes, como: serviços cooperativos, parcerias, compartilhamento e consórcio. A literatura ora os aborda como sinônimos ora destaca algumas peculiaridades que justificam uma distinção entre eles. Estamos considerando como redes, para efeito deste estudo, todos esses termos, visto que sua aplicação, seus objetivos e suas funções estão sempre relacionados ao desenvolvimento de uma atividade que deve proporcionar benefícios comuns aos seus integrantes.

De acordo com essa demarcação, uma rede de informação é tradicionalmente um grupo de unidades e serviços de informação voltado para um interesse comum, que pode ser a compilação de uma base de dados, um sistema cooperativo de catalogação, entre outras atividades, sendo seu ponto focal o compartilhamento de recursos e a cooperação em serviços e produtos. Enfim, é um arranjo formal que reúne várias organizações engajadas para a consecução de objetivos comuns, buscando a troca de informações, materiais e/ou serviços (KATZ, 1997, v.2, p.61). 
Nessa mesma linha, mas abordando outros aspectos, Vieira (1994, p.29) define rede como "[...] um conjunto de sistemas de informação e/ou comunicação - descentralizados, intercomunicantes, formados por unidades funcionais independentes, com serviços e funções inter-relacionados - cuja interação é presidida por acordos de cooperação e adoção de normas comuns".

Reiterando essas asserções, Guinchat e Menou (1994, p.340) ressaltam a legitimidade das redes, assegurando: "Estas redes devem ser formalizadas para que seus objetivos, como a repartição de tarefas e a multiplicação dos recursos, sejam atingidos plenamente. Isto significa o estabelecimento de um acordo entre os participantes e a definição de procedimentos comuns". De acordo com esses argumentos, Lozano (2004) observa que a configuração de redes de unidades de informação se constrói tendo como base um acordo de cooperação e sua principal finalidade é o intercâmbio de informações.

Referindo-se especificamente à Rede Brasileira de Informação em Ciências da Saúde, Packer (2000) destaca o crescimento e o aprimoramento dos participantes "[...] tanto individualmente como em rede. Por um lado, através da incorporação dos progressos ocorridos nas ciências da informação e nas tecnologias de informação $\mathrm{e}$, por outro lado, respondendo às novas demandas de informação em saúde". Po- demos generalizar as observações de Packer para outras redes, pois é visível a adoção de novos recursos nos últimos anos, como uma condição de sobrevivência das próprias redes.

Em relação a essa proposição, Mcgarry (1999, p.122) acredita que "A formação de redes é uma das mais importantes questões com que se defronta a comunidade bibliotecária e de informação". E isso é facilitado pelos recursos da tecnologia da informação, que incidem diretamente em todos os processos informacionais, desde sua criação e gestão até seu uso, estabelecendo-se novas formas de relacionamento entre os serviços e unidades de informação e os seus usuários, modificando seus produtos e integrando-os a outros.

Os recursos da tecnologia da informação auxiliam o desenvolvimento de processos formando bases estruturais e incorporando serviços e produtos na Internet, utilizando sua infra-estrutura para consolidar suas atividades. A Internet intensificou o compartilhamento da informação, fomentando novas redes de informação, o que acarretou o desenvolvimento de bibliotecas virtuais, digitais e eletrônicas em cooperação, bem como o compartilhamento de recursos para a formação e o desenvolvimento de coleções.

Com efeito, uma vez presentes, esses recursos facilitam o acesso à informação atualizada e relevante, propósito latente 
das redes de informação, para as quais estão direcionadas suas ações e processos, em prol dos objetivos centrais das redes.

Para compreender melhor as redes de informação, é importante conhecer seus objetivos centrais que, para Rowley (1994, p.285), permanecem constantes desde o início da década de 1970.

- mostrar o conteúdo de um grande número de bibliotecas ou de um grande número de publicações, principalmente por meio do acesso a bases de dados catalográficos, com o emprego de interfaces de catálogos em linha de acesso público;

- fazer com que os recursos mostrados nessas bases de dados catalográficos se tornem disponíveis para bibliotecas e usuários, onde e quando sejam necessários;

- compartilhar custos e esforços despendidos na criação de bases de dados catalográficos, por meio do intercâmbio de registros e atividades correlatas.

Resgatando a essência contida nos objetivos explicitados, podemos destacar: conteúdo informacional, acesso à informação, disponibilidade de registros bibliográficos, redução de custos e esforços, intercâmbio de informações. Esses elementos essenciais se harmonizam com os motivos mais comuns para o estabelecimento de redes de informação, enfatizados por Lozano (2004):

- Empréstimo entre bibliotecas;

- Organização de eventos;
- Elaboração de produtos cooperativos;

- Reunião de recursos;

- Treinamento e capacitação;

- Catalogação cooperativa;

- Consórcios para aquisição.

Os motivos que determinam a criação das redes de serviços e unidades de informação são variados e constituem o cerne das diferentes redes que têm contribuído para a ciência, a tecnologia e a educação, que só podem ser mais bem compreendidas se inseridas dentro de seu contexto histórico.

\subsection{Evolução}

As redes para serviços e unidades de informação, segundo Silva (1986, p.213), foram criadas em vista das deficiências e limitações de suas coleções, assim "a cooperação foi a solução encontrada [...] para suprir as demandas de seus usuários, ampliar os recursos informacionais e racionalizar os recursos financeiros".

A cooperação entre bibliotecas já existe desde o início do século 20 , mais precisamente, o empréstimo entre bibliotecas. Elkington e Massie (1999), fazendo uma comparação entre os serviços de empréstimos entre as bibliotecas dos Estados Unidos e as do Reino Unido, apresentam a evolução desse serviço, marcando seu inicio em 1916. Nessa época, afirmam os autores, as bibliotecas já participavam de programas cooperativos formais e infor- 
mais de empréstimo. A comunicação era feita por cartas enviadas por um mensageiro que freqüentemente levava semanas ou até meses para que todo o ciclo fosse completado e mesmo assim os pesquisadores ficavam satisfeitos.

Os serviços cooperativos foram evoluindo tornando-se mais sofisticados com o passar do tempo, principalmente após a adoção de padrões de cooperação. A catalogação cooperativa que teve seu início determinado pelo envio dos registros bibliográficos, por mensageiros, para serem completados por outra biblioteca, evoluiu; com o passar do tempo foram substituídos pelo uso do microfilme e atualmente $o$ intercâmbio é feito por formato eletrônico.

Mais tarde, em 1975, foi fundada a Research Libraries Group (RLG)5, que tinha como principal missão dar suporte ao compartilhamento de recursos entre bibliotecas. Atualmente é uma organização internacional que atua no âmbito da biblioteconomia, arquivologia e museologia, trabaIhando para criar soluções na gestão e acesso à informação. A Research Libraries Information Network (RLIN), vinculada à RLG, teve uma importante atuação no âmbito das redes, para a expansão e consolidação de produtos e serviços em parcerias. Outro organismo importante, até hoje, no âmbito dos serviços cooperativos, é a On-line Computer Library Center (OCLC)

\footnotetext{
${ }^{5}$ http://www.rlg.org/
}

que foi fundada em 1967, para o desenvolvimento da catalogação cooperativa. Elkington e Massie (1999) destacam a RLIN e a OCLC como redes que inovaram e que abriram muitos caminhos para a cooperação de serviços e unidades de informação.

Entretanto, Lozano (2004) esclarece que a primeira instituição que estruturou uma rede de informação mundial baseada na cooperação foi a Agência Internacional de Energia Atômica (IAEA - International Atomic Energy Agency - com sede em Viena - Áustria), através do Sistema Internacional de Informação Nuclear (International Nuclear Information System - INIS), que começou a operar no ano de 1970, tendo como principal motivação a produção e a disseminação de uma base de dados bibliográfica com registros do mundo todo, sobre a aplicação pacífica da ciência e tecnologia nuclear. No Brasil, seu representante é o Centro de Informações Nucleares (CIN) da Comissão Nacional de Energia Nuclear (CNEN).

Uma outra rede mundial, descrita por Lozano, que também teve início na década de 1970, mais precisamente em 1975, foi a AGRIS (Agricultural Information System) Sistema Internacional de Informação para Ciência e Tecnologia Agrícola, da Organização das Nações Unidas para a Alimentação e para a Agricultura (FAO - Food and Agriculture Organization of the United 
Nations), com sede em Roma. Para se estabelecer teve aporte da INIS/IAEA e por isso, os sistemas de suas bases de dados, nas décadas e 1970 e 1980 , eram similares, e, eram-no também seus padrões e procedimentos, isto porque a AGRIS usou inicialmente os mesmos da INIS, com pequenas modificações.

A primeira base de dados de informação bibliográfica on-line, acessível publicamente, foi a MEDLARS (Medical Literature Analysis and Retrieval System) on-line
- Medline, a partir de 1971. E o Dialog Information Services foi o primeiro maior sistema de recuperação on-line de informação do mundo - banco de dados, com importantes bases de dados.

Na América Latina, as redes mais antigas foram criadas por organismos internacionais, principalmente com o apoio do Consejo de Investigaciones e Información en Desarrollo (CIID), o Quadro 1 apresenta estas redes.

\begin{tabular}{|c|c|c|c|}
\hline Nome da Rede & Organismo Provedor & $\begin{array}{l}\text { Data da } \\
\text { criação }\end{array}$ & Área Temática \\
\hline $\begin{array}{l}\text { Centro Latino-Americano e do } \\
\text { Caribe de Informação em Ci- } \\
\text { ências da Saúde (BIREME) }\end{array}$ & $\begin{array}{l}\text { Organização Pan-americana de } \\
\text { Saúde (OPAS) }\end{array}$ & 1967 & Saúde \\
\hline $\begin{array}{l}\text { Sistema Interamericano de } \\
\text { Información } \\
\text { (AGRINTER) }\end{array}$ & $\begin{array}{l}\text { Instituto Interamericano de Coo- } \\
\text { peração para a Agricultura } \\
\text { (IICA/OEA) }\end{array}$ & 1972 & Agricultura \\
\hline $\begin{array}{l}\text { Documentos sobre Población } \\
\text { en America Latina y el Caribe - } \\
\text { DOCPAL }\end{array}$ & $\begin{array}{l}\text { Comissão Econômica para Amé- } \\
\text { rica Latina e para o Caribe } \\
\text { (CEPAL) }\end{array}$ & 1976 & População \\
\hline $\begin{array}{l}\text { Red Panamericana de Infor- } \\
\text { mación en Salud Ambiental } \\
\text { (REPIDISCA) }\end{array}$ & $\begin{array}{l}\text { Organização Pan-americana de } \\
\text { Saúde (OPAS) }\end{array}$ & 1979 & $\begin{array}{l}\text { Engenharia sani- } \\
\text { tária }\end{array}$ \\
\hline $\begin{array}{l}\text { Red de Información para el } \\
\text { Financiamiento del Desarrollo } \\
\text { (RIALIDE) }\end{array}$ & $\begin{array}{l}\text { Asociación Latinoamericana de } \\
\text { Instituciones Financieras para el } \\
\text { Desarrollo (ALIDE) } \\
\end{array}$ & 1979 & $\begin{array}{l}\text { Instituições finan- } \\
\text { ceiras }\end{array}$ \\
\hline $\begin{array}{l}\text { Sistema de Información para } \\
\text { ala Planificación en América } \\
\text { Latina y el Caribe (INFOPLAN) }\end{array}$ & $\begin{array}{l}\text { Comissão Econômica para Amé- } \\
\text { rica Latina e para o Caribe } \\
(\text { CEPAL) }\end{array}$ & 1979 & $\begin{array}{l}\text { Planejamento na } \\
\text { América Latina }\end{array}$ \\
\hline $\begin{array}{l}\text { Red Latinoamericana de Infor- } \\
\text { mación Comercial (RELIC) }\end{array}$ & $\begin{array}{l}\text { Centro de Comercio Internacional } \\
\text { del United Nations Conference on } \\
\text { Trade and Development } \\
\text { (UNCTAD/GATT) }\end{array}$ & 1981 & $\begin{array}{l}\text { Comércio e De- } \\
\text { senvolvimento }\end{array}$ \\
\hline Red Regional de CLACSO & $\begin{array}{lc}\text { Consejo } & \text { Latinoamericano } \\
\text { Ciencias Sociales (CLACSO) } & \\
\end{array}$ & 1984 & Ciências Sociais \\
\hline $\begin{array}{l}\text { Rede de Informação e Docu- } \\
\text { mentação Latino Americana } \\
\text { em Administração Pública } \\
\text { (REDLAP) }\end{array}$ & $\begin{array}{l}\text { Centro Latinoamericano de Admi- } \\
\text { nistración para el Desarrollo } \\
\text { (CLAD) }\end{array}$ & 1986 & $\begin{array}{l}\text { Administração } \\
\text { Pública }\end{array}$ \\
\hline
\end{tabular}

Fonte: Lozano (2004).

Quadro 1 - Redes de informação latino-americanas que receberam apoio da CIID

O marco inicial das redes no Brasil parece ter sido em 1942 (BARBOSA, 1978), com a catalogação cooperativa, mas é só a partir da década de 1980 que 
despontou o maior número de redes e estendeu-se a participação em redes regionais e internacionais. No Quadro 2 pode- mos observar as redes brasileiras da década de 1940 até o ano de 1982.

\begin{tabular}{|l|l|c|}
\hline \multicolumn{1}{|c|}{ Redes } & Função na época da criação & $\begin{array}{c}\text { Data da } \\
\text { Criação }\end{array}$ \\
\hline $\begin{array}{l}\text { SIC - Serviço de Intercâmbio de Catalogação, do } \\
\text { DASP }\end{array}$ & Catalogação Cooperativa & $1942-1973$ \\
\hline $\begin{array}{l}\text { CCN - Catálogo Coletivo Nacional de Publica- } \\
\text { ções Seriadas }\end{array}$ & $\begin{array}{l}\text { Formação de um catálogo cole- } \\
\text { tivo }\end{array}$ & 1954 \\
\hline $\begin{array}{l}\text { Projeto CALCO (Catalogação Legível por Com- } \\
\text { putador) - formato baseado em MARC }\end{array}$ & $\begin{array}{l}\text { Projeto para implantação da } \\
\text { Catalogação Cooperativa no no } \\
\text { Brasil }\end{array}$ & 1973 \\
\hline $\begin{array}{l}\text { Rede Brasileira de Informação em Ciência da } \\
\text { saúde - BIREME }\end{array}$ & $\begin{array}{l}\text { Serviço de Indexação e Resu- } \\
\text { mos }\end{array}$ & $\cong 1973$ \\
\hline $\begin{array}{l}\text { COMUT - Programa de Comutação Bibliográfica } \\
\text { Fornecimento de cópias de } \\
\text { documentos }\end{array}$ & 1980 \\
\hline $\begin{array}{l}\text { Rede Bibliodata CALCO - Atualmente Rede Bi- } \\
\text { bliodata }\end{array}$ & Catalogação cooperativa & 1982 \\
\hline
\end{tabular}

Quadro 2 - Principais redes no Brasil até a década de 1980

Apenas uma das redes apresentadas no Quadro 2 se extinguiu, a SIC, as outras continuam a desenvolver as funções para as quais foram criadas, em alguns casos ampliado-as.

Algumas organizações foram determinantes para a criação e crescimento das redes no Brasil. Destacamos os trabalhos do Instituto Brasileiro de Informação Científica e Tecnológica (IBICT); do Centro Latino-Americano e do Caribe em Ciências da Saúde (BIREME); e da Fundação Getúlio Vargas (FGV), que são atuantes até hoje.

\subsection{Normas e Padrões}

A inserção dos serviços e unidades de informação no âmbito das redes requer a adoção de normas e padrões que possi- bilitem o desenvolvimento de ações coadunadas, integrando diferentes organizações sob objetivos comuns.

O uso de normas e padrões na descrição dos materiais informacionais é essencial para os serviços e unidades de informação que queiram estar inseridos no contexto da cooperação nacional e internacional. A possibilidade de exportar/importar registros os insere num patamar globalizado, em que se pode considerar parte do todo.

Os padrões para importação e exportação de registros são descritos pelas normas ISO 2709 e ANSI Z39.2, que se constituem em elementos básicos para a cooperação (ZAHER, 2004). 
Além dessas normas, Zaher ressalta a importância do uso do MARC 21 para cooperação, que possibilita a gravação de registros bibliográficos, de autoridade e de fundos de todo o tipo de materiais. Ressalta, também, a adoção das regras de catalogação do AACR2 (Anglo American Cataloguing Rules) - código de catalogação anglo americano -, para a cooperação.

O formato MARC (Machine Readable Cataloging) tem como função central o intercâmbio de dados catalográficos, tornando compatíveis diferentes sistemas de informação e possibilitando o compartilhamento de recursos e a aquisição de registros bibliográficos.

O MARC 21 baseia-se na estrutura de formato ISO 2709, norma reconhecida mundialmente. Para McCallum (2004), a estrutura do MARC 21, que também é usada para o UNIMARC e para muitos outros formatos similares, é e será importante, ainda, por muitos anos. Atualmente, afirma a autora, muitos protocolos para registro bibliográfico estão surgindo com uma estrutura inteiramente baseada em XML (eXtensible Markup Language), isto é, já estão adequados para seu uso na Internet. Em resposta a essa necessidade, nesses últimos anos, têm sido desenvolvidas estruturas alternativas para os elementos de dados em MARC 21. O mais importante, segundo McCallum, é o MARCXML que pro- vê um caminho de ida e volta de MARC 21 para MARC em XML, sem prejuízos.

O desenvolvimento desses formatos, principalmente sua interação por meio de ferramentas e protocolos em XML, permite maior intercâmbio de registros em MARC 21. O uso dessas ferramentas possibilitou, conforme expõe McCallum (2004), uma transformação na Biblioteca do Congresso Americano, permitindo a pesquisa e a recuperação de informação entre computadores, operando em rede, por meio do protocolo de comunicação Z39.50 e do SRW (Search and Retrieve Web Service). A partir de então pôde ser feito o intercâmbio em vários formatos, incluindo MARC 21, MARCXML, MODS, (Metadata Object Description Standard) e Dublin Core.

O formato MODS constitui-se uma norma para descrição de objetos em metadados, desenvolvida para suprir uma necessidade do formato $\mathrm{XML}$, e o formato Dublin Core trata de um conjunto básico de elementos de metadados empregados na descrição de recursos eletrônicos, visando facilitar a sua recuperação.

A aplicação de normas e padrões em ambientes cooperativos está atrelada à evolução processual do compartilhamento e da alta qualidade do resultado final que é a razão de ser dos diferentes tipos de rede. 


\section{CATEGORIZAÇÃO DAS REDES DE INFORMAÇÃO}

O escopo e a abrangência de uma rede é que a distingue e a tipifica. Encontramos redes categorizadas: pela sua especialidade; pelo seu produto/serviço; pelo ambiente em que processa as informações - como o virtual; pelo seu âmbito - espaço em que atua - nacional, regional, internacional; entre outras categorizações.

Alguns tipos de redes, que também podem ser denominadas de redes de informação, estão presentes em universidades e em comunidades científicas, sem ter, necessariamente, a parceria de um serviço ou uma unidade de informação, tais como: listas de discussão, comunidades virtuais, ciberfóruns e outros recursos de comunicação e compartilhamento possibilitados pela Internet, que não são objeto de estudo deste trabalho, que têm seu foco nas redes formais de informação em que participam serviços e unidades de informação, apesar de muitas das redes de informação, arroladas nesta pesquisa, as utilizarem como recurso para a comunicação entre seus membros.

Para compreendermos melhor a categorização das redes de informação apresentamos as abordagens de Guinchat e Menou (1994), Katz (1997) e Vieira (1994), denominando-as e descrevendo-as pelas suas funções e suas características.
De acordo com a função das redes, Guinchat e Menou (1994, p.340) as distinguem categorizando-as em diferentes tipos que, segundo eles, podem ser combinados entre si.

- redes especializadas em funções documentais, como a aquisição, o tratamento de documento (catalogação, classificação, análise e indexação), e a difusão (empréstimo, comutação bibliográfica, difusão seletiva da informação e serviço de pergunta e resposta);

- redes que integram as unidades participantes em um sistema de informação único que cobre todas as funções documentais. [...]

- redes enciclopédicas ou redes especializadas em uma disciplina ou em um ramo de atividade, nas quais todas as unidades de informação associam-se para apoiar-se mutuamente ou para harmonizar seus serviços e seus produtos;

- redes especializadas a serviço de uma categoria particular de usuários, como as pequenas empresas ou os produtores de café, por exemplo.

A especialização tem papel determinante na tipologia proposta pelos autores; aparece em três dos quatro tipos categorizados, empregada distintamente, a saber: a) função da rede, mais propriamente pelos serviços desenvolvidos; b) especialidade disciplina; c) usuário dos serviços/produtos da rede.

Diferentemente de Guinchat e Menou, Katz (1997, v.2, p.61) categoriza as redes de serviço em dois tipos: rede comercial que visa lucro e a rede que não visa lucro. Uma processa a informação, enquanto a 
outra comunica dados para seus usuários. Alguns sistemas combinam ambas.

As redes que não visam lucro são denominadas pelo autor de redes bibliográficas ou de serviços bibliográficos. Essas redes podem ser regionais, estaduais, nacionais ou ainda internacionais e possibilitam acesso a catálogos de várias bibliotecas ao mesmo tempo (catálogos coletivos) ou ao catálogo de uma biblioteca em especial. As redes, em questão, provêem serviços diversos para tratamento e recuperação da informação, como os que respondem a questões de referência em que se buscam, localizam e completam informações. As maiores redes bibliográficas dessa categoria, segundo o autor, são a Online Computer Library Center (OCLC) e a Research Libraries Information Network (RLIN).

A OCLC 6 é uma organização dedicada à pesquisa e à organização de serviços bibliotecários, com o propósito de promover o acesso à informação do mundo todo a custos reduzidos. Participam dessa rede mais de 50 mil bibliotecas em 84 países e territórios por todo o mundo, usando serviços da rede para localizar, adquirir, catalogar, emprestar e preservar materiais bibliográficos.

A RLIN ${ }^{7}$ é um sistema de informação bibliográfico disponível internacionalmente que subsidia os processos de catalogação, além de outros serviços que compreendem o empréstimo entre bibliotecas e a aquisição.

O outro tipo de rede comercial, apresentada por Katz, é a de processamento e distribuição da informação. Nessa rede a informação é vendida para indivíduos ou para organizações, são comerciais e visam lucro. Entre as exemplificadas pelo autor está o Dialog Information Services.

O Dialog Information Services ${ }^{8}$, com centenas de bases de dados, é o melhor serviço on-line conhecido. É líder mundial no provimento de serviços on-line para organizações que procuram vantagens competitivas, incorporando bases de dados das áreas: negócios, ciência, engenharia, finanças e direito.

Incluímos nessa categoria, também, o STN International ${ }^{9}$ - Scientific \& Technical Information Network, rede de informação científica e tecnológica que é produzida cooperativamente, por FIZ Karlsruhe - Alemanha, pelo American Chemical Society (ACS) por meio do Chemical Abstracts Service (CAS) - Columbus - Ohio - Estados Unidos, e pelo Japan Science and Technology Corporation (JST) - Tókio - Japão, há cerca de 15 anos e provê serviços de informação para o mundo todo, disponibilizando um banco de dados, com mais de

\footnotetext{
${ }^{6} \mathrm{http}: / /$ www.oclc.org

${ }^{7}$ http://www.rlg.org/rlin.html
} 
200 bases, nas áreas de: energia, física, matemática, química, ciência, tecnologia e medicina.

Uma outra distinção dos tipos de redes que merece destaque é a de Vieira (1994, p.31-51) que categoriza as redes em três grupos consoantes com as funções a que se propõem:

a) Redes de comunicação de dados: "consistem em um conjunto de computadores conectados por recursos da telemática, para o transporte de dados e mensagens entre dois pontos distantes interligados". Os serviços mais utilizados nestas redes são: correio eletrônico, transferência de arquivo/software, chat, listas de discussões e WWW. Essa categorização não está vinculada diretamente à abordagem de redes de informação que nos propusemos a estudar e apresentar neste trabalho, mas certamente constitui-se um recurso imprescindível para elas.

b) Redes de serviços e de apoio institucional a sistemas de informação, "tem como objetivo colaborar no desenvolvimento de padrões comuns, na organização dos registros bibliográficos e no intercâmbio (de dados e documentos) entre bibliotecas ou centros de informação". Exemplos desse tipo de rede são representados pela catalogação cooperativa e pelo empréstimo entre bibliotecas.
Vieira destaca a participação brasileira nas redes internacionais dessa categoria que são: ISDS (International Serials Data System), atualmente denominada Rede ISSN - International Standard Serial Number - (ISSN Network), e RITLA (Red de Información Tecnológica Latino-americana).

As nacionais que mereceram destaque da autora são: Bibliodata, rede de catalogação cooperativa, gerida pela Fundação Getúlio Vargas; Comut (Programa de Comutação Bibliográfica), sediado no IBICT, proporciona acesso ao documento primário; $\mathrm{CCN}$ que tem como objetivo compilar uma base de dados que disponibilize informações referente às coleções de periódicos nacionais e estrangeiros de todas as bibliotecas cooperantes.

c) Redes de informação especializada a usuários, "dedicam-se ao fornecimento de informações (dados bibliográficos, factuais, cadastrais, etc.) e documentos diretamente ao usuário final". O acesso a essas redes é feito através de sistemas online.

Segundo Vieira, as principais redes internacionais de informações especializadas que têm a participação brasileira são: AGRIS; Infoterra, INIS, MEDLARS e REPIDISCA. Citamos, aqui, as que foram contempladas no levantamento que deu origem a este trabalho. 
Quanto às nacionais, Vieira seciona o mercado em três grupos: a) segmento técnico-científico - cientistas, tecnólogos e gestores de Ciência e Tecnologia. b) segmento industrial-empresarial - empresários e industriais de pequeno e médio porte. c) segmento leigo alternativo - cidadãos leigos, pequenos agricultores e administradores municipais: informações sobre tecnologias alternativas.

A conjunção dos tipos de redes apresentados, aliados à análise das identificadas por este trabalho, nos permitiu fazer uma nova categorização que resultou em cinco tipos de redes:

Redes de Compatibilização da Informação (RCI): incluem serviços e unidades de informação que reúnem seus catálogos formando catálogos coletivos. O produto resultante do trabalho cooperativo é multidisciplinar e consolida a principal função da rede. Usualmente são utilizados para a localização de documentos.

Redes de Processamento da Informação (RPI): compreendem as redes que organizam a informação, envolvendo processos de descrição e indexação da informação - como a catalogação cooperativa , normalmente disponibilizam catálogos coletivos ou bases de dados bibliográficas multidisciplinares. Sua principal função está direcionada a apoiar os serviços e unidades de informação em suas atividades de organização da informação, subsidiando sistemas de gerenciamento de coleções.

Redes de Serviços de Informação (RSI): pertencem a essa categoria redes constituídas por serviços e unidades de informação que prestam serviços recíprocos e para clientes isolados ou para comunidades específicas, envolvendo suas coleções e seus especialistas nesse esforço. Algumas utilizam produtos resultantes das redes de processamento da informação, como instrumentos para a consecução de suas atividades.

\section{Redes de Informação Especializada} (RIE): fazem parte dessa categoria redes que tratam de um ramo específico, dentro de uma área do conhecimento, e desenvolvem atividades diferenciadas, o maior número, dessas redes, opera na organização da informação, principalmente por meio dos serviços de indexação e resumos, mas há redes que tratam, prioritariamente, do intercâmbio de cópias de documentos. Habitualmente, disponibilizam bases de dados bibliográficas como produto final da rede.

\section{Redes de Informação Digital (RID):} distinguem-se por utilizarem amplamente os recursos da Internet. Na maior parte dos casos apresenta a informação propriamente, não apenas sua indicação. 


\section{REDES DE INFORMAÇÃO NO BRASIL $^{10}$}

Por meio de 40 sites na Internet de bibliotecas universitárias e pela análise das primeiras 50 indicações do Google, para quatro pesquisas distintas, identificamos 41 redes que têm a participação de serviços e unidades de informação do Brasil.

Dessas 41 redes, três atuam em âmbito estadual; 10 regionalmente, principalmente na América Latina e no Caribe; 20 atingem toda a nação - redes nacionais e oito têm alcance internacional.

A partir das cinco categorizações definidas neste trabalho - redes de compatibilização da informação (RCl); redes de processamento da informação (RPI); redes de serviços de informação (RSI); redes de informação especializada (RIE); redes de informação digital (RID) - classificamos as redes identificadas por este estudo, pela sua principal motivação.

\subsection{Redes de Compatibilização da In- formação (RCl)}

A compatibilização de catálogos resulta em catálogos coletivos que, além de serem agentes do controle bibliográfico e da recuperação da informação, são poderosos instrumentos para serviços de obtenção e organização de documentos.

\footnotetext{
${ }^{10}$ As informações apresentadas do item 4.1 ao item 4.5, foram compiladas a partir das informações disponíveis nos sites das redes e das organizações membros e as URLs (Universal Resource Locator) consultadas estão indicadas no decorrer da apresentação das redes.
}

Nessa categoria estão inseridas três redes nacionais, uma regional e duas internacionais.

\section{a) Redes Nacionais}

- Catálogo Coletivo Nacional de Publicações Seriadas (CCN) - Multidisciplinar ${ }^{11}$

- Ação central: União de catálogos. Produto: Catálogo Coletivo

- Possibilita a reunião de catálogos de publicações periódicas, nacionais e estrangeiras, das principais bibliotecas do Brasil, em um catálogo coletivo de acesso público. Tem o objetivo de difundir, identificar e localizar as "publicações seriadas em C\&T, nacionais e estrangeiras, existentes no país"; estabelecer políticas de aquisição cooperativa; e padronizar entradas.

- Catálogo Coletivo de Normas Técnicas Multidisciplinar $^{12}$

- Ação central: União de catálogos. Produto: Catálogo Coletivo

- Rede cooperativa formada por bibliotecas brasileiras que culmina em uma base de dados bibliográfica que contém normas técnicas nacionais e estrangeiras. Tem o objetivo de disseminar normas técnicas disponíveis nas bibliotecas que integram a rede.

- Rede Compartilhada do Sistema Pergamum - Multidisciplinar ${ }^{13}$

- Ação central: União de catálogos. Produto: Catálogo Coletivo.

- O pergamum é um sistema de gerenciamento de bibliotecas. Seu objetivo é "Aproveitar as principais idéias de cada instituição a fim de manter o software atualizado e atuante no mercado, tornando-o capaz de gerenciar qualquer tipo de documento", atendendo todo tipo de biblioteca.

\footnotetext{
${ }^{11} \mathrm{http}: / /$ www.ibict.br/ccn/inicio.htm

12 http://cin.cnen.gov.br/catalogos/busca/busca.html

${ }^{13}$ http://www.pergamum.pucpr.br
} 


\section{b) Rede Regional}

- Catálogo Coletivo de Anais de Eventos - Multidisciplinar ${ }^{14}$

- Ação central: união de catálogos. Produto: Catálogo coletivo.

- Rede cooperativa consolidada em um catálogo bibliográfico que contém todo tipo de anais de eventos realizados no Brasil e na América Latina, em diversas áreas do conhecimento. Seu objetivo é disseminar anais de eventos por meio da base de dados anais.

c) Redes Internacionais

- WorldCat - Multidisciplinar ${ }^{15}$

- Ação central: união de catálogos. Produto: Catálogo coletivo.

- A rede WorldCat forma a maior base de dados bibliográfica do gênero do mundo, promovendo a união de catálogos de milhares de bibliotecas membros do OCLC. Serve de base para muitos serviços da OCLC. Tem o objetivo de servir de instrumento para catalogadores da rede OCLC.

- Rede ISSN - International Standard Serial Number - (ISSN Network) - Multidisciplinar ${ }^{16}$

- Ação central: Controle de publicações seriadas. Produto: Catálogo coletivo.

- Organização intergovernamental, implantada em 1974. O ISSN - Número Internacional Normalizado para Publicações Seriadas - identifica o título de uma publicação seriada; é aceito internacionalmente. Tem o objetivo de "Apoiar o controle bibliográfico mundial de publicações seriadas, facilitando o acesso aos registros e controlando a atribuição do ISSN".

\subsection{Redes de Processamento da Infor- mação (RPI)}

$\mathrm{O}$ que move as redes agrupadas nessa categoria é a catalogação cooperativa,

\footnotetext{
${ }^{14}$ http://cin.cnen.gov.br/catalogos/busca/busca.html

${ }^{15} \mathrm{http}: / /$ www.oclc.org/worldcat/default.htm

${ }^{16}$ http://www.issn.org:8080/English/pub/network
}

que visa facilitar e agilizar os processos de descrição bibliográfica de forma cooperativa, tornando esses processos e o intercâmbio de registros possíveis, através da adoção de normas e padrões que os possibilitam de fato.

Essa categoria compreende três redes, duas nacionais e uma internacional.

\section{a) Redes Nacionais}

- Rede Bibliodata - Multidisciplinar ${ }^{17}$

- Ação central: Catalogação cooperativa. Produto: catálogo coletivo.

- Rede formada por bibliotecas brasileiras. Tem como principal função a catalogação cooperativa. Seu objetivo é "Desenvolver e manter o Catálogo Coletivo da Rede, desenvolver metodologias e instrumentos para a catalogação cooperativa, gerando subsídios para o compartilhamento de serviços e recursos entre as instituições participantes".

- Consórcio Eletrônico de Bibliotecas Multidisciplinar ${ }^{18}$

- Ação central: Catalogação cooperativa.

- Criado em 1999, para o intercâmbio de registros bibliográficos, pela Internet, das bases da Fundação Biblioteca Nacional, disponíveis no site: http://www.bn.br. Tem como objetivo "Apoiar o desenvolvimento dos projetos de automação bibliográfica no Brasil, permitindo às bibliotecas, através do compartilhamento dos recursos de catalogação on-line da Biblioteca Nacional, a formação de bases de dados locais ou de redes de bases regionais".

b) Rede Internacional

- Program for Cooperative Cataloging PCC (Programa de Catalogação Coo-

\footnotetext{
${ }^{17}$ http://www.fgv.br/bibliodata

${ }^{18}$ http://consorcio.bn.br/consorcio/
} 
perativa) - Multidisciplinar ${ }^{19}$ - NACO / Library of Congress - EUA (cooperação técnica de manutenção de base de dados de padronização internacional de autoria)

- Ação central: Catalogação cooperativa. Produto: Catálogo de autoridade.

- Programa de cooperação internacional, excelente indicador de cooperação em catalogação, que visa expandir $\mathrm{o}$ acesso a registros bibliográficos, possibilitando a rapidez e o baixo custo da catalogação mediante o emprego de padrões aceitos internacionalmente. Objetivo: "Aumentar e agilizar, por meio de ações cooperativas, a disponibilidade de registros bibliográficos e de autoridades, produzindo catálogos a serem usados e compartilhados em outras instituições, a um custo controlado".

\subsection{Redes de Serviços de Informação (RSI)}

Essa categoria contém redes que prestam apoio a serviços e unidades de informação, principalmente por meio dos serviços de empréstimo entre bibliotecas e comutação. Inserem-se nessa categoria, também, as redes que dão apoio às microempresas e as pequenas empresas, desenvolvendo serviços de informação que promovam a inovação e a competitividade empresarial.

Primeiramente apresentamos as redes que apóiam os serviços e unidades de informação, que abrangem duas redes estaduais, uma regional e uma internacional. Em seguida identificamos as redes que apóiam às microempresas e as pequenas

\footnotetext{
${ }^{19}$ http://authorities.loc.gov/cgibin/Pwebrecon.cgi?DB=local\&PAGE=First
}

empresas, que fornecem, principalmente, serviços de informação tecnológica. Estas compreendem uma rede estadual, duas nacionais e uma regional.

\subsubsection{Serviços e Unidades de Informa- ção}

Poucas são as bibliotecas que conseguem atender seus usuários servindo-se apenas de suas coleções. Rodrigues (2004), fazendo referência ao Brasil, comenta a baixa disponibilidade de documentos nas bibliotecas, devido a restrições financeiras. Para o autor, a falta de qualidade de muitos acervos é suprida pela utilização conjunta de acervos de diferentes bibliotecas e serviços de informação de forma cooperativa.

O serviço de comutação bibliográfica permite a um usuário ou a uma organização/biblioteca a obtenção de cópias de documentos que não detém em sua biblioteca.

\section{a) Redes Estaduais}

- Compartilhamento Entre Bibliotecas de Instituições de Ensino Superior do Estado do Rio de Janeiro - Multidisciplinar $^{20}$

- Ação central: Intercâmbio de documentos.

- Intercâmbio do acervo das bibliotecas das instituições públicas e privadas e instituições de ensino e pesquisa (associadas). Objetivo: Promover o intercâmbio para o uso dos acervos e do conhecimento científico, tecnológico e acadêmico entre as instituições participantes. "Esta-

\footnotetext{
${ }^{20}$ http://www.ndc.uff.br/compartilhamento/
} 
belecer relações com organizações e entidades nacionais e internacionais que possam fornecer recursos". - CRUESP/Bibliotecas - Multidisciplinar ${ }^{21}$

- Ação central: Intercâmbio de documentos. Produto: Catálogo coletivo.

- Compartilhamento de informação mediante reunião dos sistemas de bibliotecas da USP, UNICAMP e UNESP, tendo como serviços principais: o acesso a bases de dados pelo Portal de Pesquisa ${ }^{22 .}$ Objetivo: "Criar condições para o funcionamento sistêmico das bibliotecas [...] e a otimização dos recursos destinados às Bibliotecas, a fim de oferecer suporte ao desenvolvimento do ensino e pesquisa".

\section{b) Rede Regional}

- Programa de Comutação Bibliográfica Comut - Multidisciplinar ${ }^{23}$

- Ação central: Intercâmbio de documentos.

- O Comut é uma rede estruturada para o fornecimento de cópias de documentos como: periódicos, teses, anais de congressos, e partes de outros documentos. Dentre os objetivos do Comut ressaltamos o de "Facilitar o acesso ao documento requerido nas tarefas de pesquisa, ensino e gerenciamento independentemente de sua localização".

\section{c) Rede Internacional}

- British Library - Document Supply Centre - BLDSC (Comutação Internacional) - Multidisciplinar ${ }^{24}$

- Ação central: Intercâmbio de documentos. Produto: Catálogo coletivo.

- Fornecimento de documentos e serviço de empréstimo entre bibliotecas, provavelmente é o maior serviço de comutação do mundo. Tem como suporte a coleção da Biblioteca Britânica que cobre todos os aspectos do conhecimento científico,

\footnotetext{
${ }^{21}$ http://bibliotecas-cruesp.usp.br/search.html

${ }^{22}$ Ver a rede "Portal de Pesquisa" categorizada em Redes de Informação Digital (RID)

${ }^{23} \mathrm{http}: / / 200.214 .187 .5: 8080 /$ comut/do/index?op=filtroForm

${ }^{24} \mathrm{http}: / /$ www.bl.uk/services/document/dsc.html
}

técnico, médico e humano, em muitas línguas.

\subsubsection{Empresas}

\section{a) Rede Estadual}

- RITEC - Rede de Inovação e Tecnologia do Paraná - Multidisciplinar ${ }^{25}$

- Ação central: Apoio a inovação e a competitividade industrial. Serviço: Resposta Técnica.

- Rede que tem como foco o setor produtivo, contempla a informação tecnológica e a extensão tecnológica. Constituindo-se um programa de atendimento às demandas tecnológicas das empresas paranaenses. Objetivo: Subsidiar informações tecnológicas às microempresas e às pequenas empresas paranaenses.

\section{b) Redes Nacionais}

- RETEC - Rede SENAI/IEL de Tecnologia - Multidisciplinar ${ }^{26}$

- Ação central: Apoio à inovação e a competitividade industrial. Serviços: Informação e Extensão tecnológica.

- A rede iniciou suas atividades na Bahia, em 2001, e atualmente vem sendo replicada para: Distrito Federal, Minas Gerais, Paraná, Ceará e Amazonas, isto é, a rede é constituída por unidades estaduais. Objetivo: Disponibilizar o acesso à informação e ao conhecimento aos microempresários e aos pequenos empresários, para fortalecer seu crescimento e sua competitividade industrial.

- Sistema Brasileiro de Resposta Técnica - SBRT - Multidisciplinar ${ }^{27}$

- Ação central: Apoio a inovação e a competitividade industrial. Serviço: Resposta técnica.

- Resposta técnica é um serviço especializado que, por meio da literatura técnico-científica e de especialistas, propõe soluções para diferentes tipos de questões empresariais. $O$ objetivo da rede é buscar soluções

\footnotetext{
${ }^{25}$ http://spert.ritec.tecpar.br/

${ }^{26} \mathrm{http}: / /$ www.retec.org.br

${ }^{27}$ http://sbrt.ibict.br/
} 
para problemas tecnológicos apresentados pelo setor produtivo, promovendo o acesso ao conhecimento e contribuindo para a transferência de tecnologia.

\section{c) Rede Regional}

- RITLA - Rede de Informação Tecnológica Latino-Americana (em inglês a sigla é LATIN) - Multidisciplinar ${ }^{28}$

- Ação central: apoio à pequena e microempresa. Produto: Infra-estrutura de informação.

- Organismo internacional intergovernamental criado em 1983 no campo de ação do Sistema Econômico Latino-Americano - SELA. Objetivo: Impulsionar o desenvolvimento da informação, por meio da cooperação científica e tecnológica, na América Latina e no Caribe, apoiando infraestruturas e sistemas de informação dos estados membros.

\subsection{Redes de Informação Especializada (RIE)}

São redes que atuam no âmbito da informação especializada, em campos específicos do conhecimento, em que a maioria delas se dedica aos serviços de indexação e resumos que produzem as bases de dados, mas há, também, redes que desenvolvem: empréstimo entre bibliotecas, comutação, bases cadastrais, instrumentos terminológicos, entre outros.

Participam dessa categoria seis redes nacionais, sete regionais e quatro internacionais.

\section{a) Redes Nacionais}

- Rede Brasileira de Bibliotecas da Área de Psicologia - ReBAP - Psicologia ${ }^{29}$.

\footnotetext{
${ }^{28}$ http://www.ritla.net/

${ }^{29}$ http://www.bvs-psi.org.br/rebap
}

- Ação central: Serviço de indexação e resumos. Produto: Biblioteca virtual.

- Possibilita o acesso à informação, para o ensino, pesquisa e práticas psicológicas, visando o desenvolvimento da Psicologia no Brasil. Objetivo: "Operar de forma integrada, buscando o compartilhamento de recursos e a cooperação de esforços, com vistas à promoção do acesso eficiente e eqüitativo à informação e ao documento ao profissional e estudioso da Psicologia, independente da região do País".

- Rede Brasileira de Informação em Ciências da Saúde ${ }^{30}$ - Ciências da Saú$\mathrm{de}^{31}$.

- Ação central: Serviço de indexação e resumos. Produto: Biblioteca virtual.

- Desenvolve um trabalho cooperativo no Brasil, há mais de 30 anos, provendo o acesso à informação técnico-científica para pesquisadores e profissionais da área da saúde. Objetivo: "Promover o acesso eqüitativo à informação em saúde".

- Rede de Bibliotecas na Área de engenharia - REBAE - Engenharia ${ }^{32}$.

- Ação central: Serviço de indexação e resumos. Produto: Diretório de bases de dados.

- Rede de cooperação na área de Engenharia. Tem como atribuição identificar, reunir e organizar informações na área de engenharia visando a cooperação. Objetivo: "Facilitar o acesso à informação e ao documento, no Brasil e exterior".

- Rede Nacional de Informações em Saúde - RNIS - Ciências da Saúde ${ }^{33}$.

- Ação central: Apoio à gestão em saúde. Produto: Cadastro de instituições.

\footnotetext{
${ }^{30}$ Informações baseadas nos sites http://www.bireme.br, http://saudepublica.bvs.br e no texto de Packer (2000).

${ }^{31} \mathrm{http}: / / \mathrm{www}$.bireme.br

32 http://www.cnptia.embrapa.br/biblio/rebae/

${ }^{33} \mathrm{http}$ ://www.datasus.gov.br/rnis/datasus.htm
} 
- Utiliza a Internet para integrar municípios brasileiros, possibilitando o acesso e o intercâmbio de informações em saúde. Objetivo: "Integrar e disseminar as informações de saúde no país e contribuir para a melhoria da gestão, do controle social, do planejamento e da pesquisa de gestores, agentes e usuários do Sistema Único de Saúde (SUS)".

- Sistema Brasileiro de Documentação e Informação Desportiva - SIBRADID Esporte e Educação Física ${ }^{34}$.

- Ação central: Serviço de indexação e resumo. Produtos: Bases de dados.

- Seu principal propósito é a formação de bases de dados cooperativa, visando o acesso e a obtenção de informações disponíveis nos centros cooperantes. Seu objetivo é "promover e disseminar o uso das informações contidas nas Bases de Dados do Sistema, possibilitando a prestação de serviços de acesso a documentos através da Comutação Bibliográfica".

- RENIMA - Rede Nacional de Informação sobre Meio Ambiente - Meio Ambiente ${ }^{35}$.

- Ação central: Serviço de indexação e resumo. Produtos: Bases de dados.

- Criada em 1993 para fortalecer as unidades participantes através da capacitação de recursos humanos, mobiliário e acervo bibliográfico. Objetivo: "Dar suporte informacional às atividades técnico-científicas e industriais e apoiar o processo de gestão ambiental".

\section{b) Redes Regionais}

- Interligação de Bibliotecas para Troca de Documentos - LIGDOC - do IberoAmerican Science and Technology E- ducation Consortium - ISTEC - Engenharia ${ }^{36}$.

- Ação central: Intercâmbio de documentos. Produto: Catálogo coletivo.

- Consórcio, patrocinado pelo ISTEC, para o intercâmbio de documentos entre bibliotecas ibero-americanas, desenvolvido pela Universidade do Novo México (EUA). Objetivo: Possibilitar o intercâmbio de documentos, pela Internet, para a promoção científica e tecnológica dos países membros.

- Red Latinoamericana de Información Y Documentación en Educación REDUC - Educação ${ }^{37}$.

- Ação central: Serviço de indexação e resumo. Produto: Bases de dados.

- Informação especializada em áreas temáticas prioritárias da educação latino-americana. Objetivo: Vincular o conhecimento em educação com as ações e os processos de tomada de decisões, procurando contribuir para a melhoria da qualidade e eficiência da educação dos países da região.

- Serviço Cooperativo de Acesso ao Documento da Biblioteca Virtual em Saúde - SCAD - Ciências da Saúde ${ }^{38}$.

- Serviço de comutação de fotocópias de documentos, no qual as bibliotecas cooperantes disponibilizam suas coleções para outras unidades da rede. Objetivo: "Prover o acesso a documentos da área de Ciências da Saúde, exclusivamente para fins acadêmicos e de pesquisa".

- Latin American Periodicals Tables of Contents - LAPTOC - Ciências Sociais e Humanas ${ }^{39}$.

- Ação central: Serviço de indexação e resumos. Produto: Base de dados bibliográfica.

\footnotetext{
${ }^{34} \mathrm{http}: / / w w w . s i b r a d i d . e e f . u f m g . b r$

${ }^{35}$ http://www2.ibama.gov.br/ cnia/renima.htm
} 
- Base de dados disponível na Web que provê o acesso aos sumários de mais de 800 periódicos, principalmente nas Ciências Sociais e Humanas publicados na América Latina, propiciando seu acesso por meio das bibliotecas cooperantes. Objetivo: Propiciar o acesso a informações de periódicos latino-americanos.

- Rede Pan-Americana de Informação em Saúde Ambiental - REPIDISCA Meio ambiente.

- Ação central: Serviço de indexação e resumos. Produto: Bases de dados.

- Seu foco centra-se na seleção e análise de materiais bibliográficos, como: pesquisas, informes técnicos, teses, anais de eventos, normas técnicas, vídeos programas de computador, e materiais didáticos. No Brasil o coordenador é o Bireme. Objetivo: Difundir informação na área de especialidade.

- Sistema Latino-Americano e do Caribe de Informação em Ciências da Saúde Ciências da Saúde ${ }^{40}$.

- Ação central: Serviço de indexação e resumos. Produto: Bases de dados.

- Criação cooperativa de bases de dados locais, nacionais e regionais. Objetivo: Contribuir para o controle bibliográfico e para a disseminação da literatura médica da região.

- Sistema de Información y Documentación Agropecuario de las Américas SIDALC - Agropecuária ${ }^{41}$.

- Ação central: Serviço de indexação e resumos. Produto: Bases de dados.

- Tem como objetivo facilitar a troca de informação e possibilitar o acesso à literatura agropecuária da América Latina e do Caribe.

\section{c) Redes Internacionais}

- International Nuclear Information System - INIS - Energia Nuclear ${ }^{42}$.
- Ação central: Serviço de indexação e resumos. Produto: Base de dados bibliográfica.

- A base de dados bibliográfica INIS é o principal produto do sistema cooperativo. O acesso à base para o Brasil é propiciado pela CNEN, que é o representante do Brasil no INIS.

- Infoterra - The Global Environmental Information Exchange Network - Meio Ambiente ${ }^{43}$.

- Ação central: Serviço de indexação e resumos. Produto: bases de dados.

- Rede mundial para intercâmbio de informação ambiental científica e técnica. Objetivo: Disponibilizar informação e fortalecer os centros coordenadores nacionais, reorganizando seus ambientes informacionais.

- AGRIS - Agricultural Information System - Agricultura ${ }^{44}$.

- Ação central: Serviço de indexação e resumos. Produto: Bases de dados.

- Sistema cooperativo internacional de informação para a ciência e tecnologia agrícola. Foi criado pela FAO em 1974. Tem como objetivo facilitar a troca de informação e possibilitar o acesso à literatura mundial em agricultura.

- MEDLARS - Medical Literature Analysis and Retrieval System - Ciências Médicas $^{45}$.

- Ação central: Serviço de indexação e resumos. Produto: Bases de dados.

- Sistema de processamento e recuperação da informação médica, biomédica, odontológica, enfermagem, veterinária e ciências afins. Seu objetivo é organizar e disponibilizar informação em Ciências Médicas.

\footnotetext{
${ }^{40}$ http://www.bireme.br

${ }^{41}$ http://orton.catie.ac.cr

42 http://cin.cnen.gov.br/inis-brasil/
}

\footnotetext{
${ }^{43}$ http://www.unep.org/infoterra

${ }^{44} \mathrm{http}: / / w w w . f a o . o r g / a g r i s$

${ }^{45}$ http://www.nlm.nih.gov/
} 


\subsection{Redes de Informação Digital (RID)}

Estão classificadas nessa categoria redes que processam e reúnem a informação disponível na Internet, além de redes que possibilitam o acesso ao texto completo da informação científica e tecnológica. Incluímos nessa categoria, também, redes que promovem a reunião personalizada de bases de dados, bibliográficas ou de texto completo, de acordo como interesse do usuário. As sete redes aqui agrupadas são todas nacionais.

- Biblioteca Digital Brasileira - BDB Multidisciplinar $^{46}$.

- Ação central: texto completo. Produto: Biblioteca digital.

- Sistema cooperativo que funciona integralmente na Internet e se propõe a integrar diversos repositórios de informação digital em um único portal. Objetivo: Estar atento às exigências e necessidades de serviços de informação inovadores. "Contribuir para aumentar o acesso aos documentos eletrônicos que sejam de interesse para o desenvolvimento das atividades técnicas e cientificas, assim como para os demais setores importantes para o desenvolvimento econômico e social do país, tais como o de educação e o produtivo".

- Portal de Periódicos da CAPES - Portal Brasileiro da Informação Científica Multidisciplinar ${ }^{47}$.

- Ação central: Texto completo. Produto: Portal de periódicos.

- Serviço oferecido pela CAPES que possibilita o acesso imediato a bases de dados referenciais e a textos completos de artigos da produção científica. Objetivo: "Promover a elevação da qualidade do ensino supe-

\footnotetext{
${ }^{46}$ http://www.ibict.br/bdb/inicio.htm

${ }^{47}$ http://www.periodicos.capes.gov.br/
}

rior através do fomento a pósgraduação".

- Portal da Pesquisa - Multidisciplinar ${ }^{48}$.

- Ação central: Acesso a bases de dados. Produto: Bases de dados.

- Produzido pela "DotLib Informação profissional"

(http://www.dotlib.com.br/), empresa especializada no fornecimento de bases de dados e publicações eletrônicas para o setor acadêmico e de pesquisa. Objetivo: Criar bibliotecas virtuais e websites personalizados de acordo com o interesse da instituição conveniada.

- Programa Prossiga - Portal de ICT Prossiga - Multidisciplinar ${ }^{49}$.

- Ação central: Acesso à informação na Internet. Produto: Portal de Informação científica e tecnológica.

- Atua na área de ICT, criando meios de acesso à informação brasileira. Objetivo: "promover a criação e o uso de serviços de informação na Internet voltados para as áreas prioritárias do Ministério da Ciência e Tecnologia, assim como estimular o uso de veículos eletrônicos de comunicação pelas comunidades dessas áreas".

- Páginas Brasileiras - Multidisciplinar ${ }^{50}$.

- Ação central: Acesso à informação na Internet. Produtos: Localizador.

- Portal de informações brasileiras em Ciência, Tecnologia e Educação, integrado ao Programa Prossiga. Está presente nos estados brasileiros através de instituições parceiras que colaboram na reunião e disponibilização das informações pertinentes a sua área geográfica. Tem como objetivo localizar informações em sites, que não estejam contempladas nos sistemas formais de informação em Ciência, Tecnologia e Educação.

- RENAI - Rede Nacional de Informações sobre Investimento - Economia ${ }^{51}$.

\footnotetext{
${ }^{48}$ http://www.portaldapesquisa.com.br/databases/sites

49 http://www.prossiga.br/

http://www.prossiga.br/informacaoct/

50 http://www.prossiga.br/paginasbrasileiras/
} 
- Ação central: Acesso à informações na Internet. Produto: Portal de informações.

- Tem como foco a atividade de investimento no Brasil e seu objetivo constitui-se em disponibilizar informações direcionadas a investidores, entidades de fomento do desenvolvimento, órgãos de pesquisa, organismos públicos, etc.

- Cadê Jur - Direito ${ }^{52}$.

- Ação central: Informação na Internet. Produto: Portal de informação.

- Portal jurídico que possibilita a localização de advogados, escritórios de advocacia, estudantes de direito, magistrado, membros do ministério público, peritos, professores e produtos e serviços da área jurídica. Tem o objetivo de promover o intercâmbio de informações, proporcionando a troca de experiências.

\section{CONSIDERAÇÕES FINAIS}

As redes de informação no Brasil estão consolidando ações que se concentram em funções que vão desde as tradicionais, como já se fazia no início do século 20 , até as essencialmente produzidas em ambiente virtual. As principais ações/atividades das redes concentram-se em: Intercâmbio de materiais informacionais (empréstimo entre bibliotecas; comutação de cópias de documentos e arquivos); União de catálogos; Catalogação cooperativa; Serviço de indexação e resumos; Acesso ao texto completo de documentos em Ciência e Tecnologia; Acesso a informações disponí-

\footnotetext{
${ }^{51} \mathrm{http}: / /$ sistemasweb.desenvolvimento.gov.br/INVES TIMENTO_WEB

52 http://www.cadejur.com.br/
}

veis na Internet; Apoio informacional à inovação e à competitividade industrial.

Para executar essas funções, as redes de informação estão pondo em prática seus objetivos, que se constituem em alvos a serem atingidos, por meio das ações implementadas pelas redes. Analisando os objetivos das redes verificamos que eles estão concentrados em quatro dimensões.

$\mathrm{Na}$ primeira dimensão encontramos objetivos relacionados ao acesso à informação dispersa em diferentes materiais informacionais e localidades, e consistem em: Identificar e localizar materiais informacionais; Promover o intercâmbio de informações; Impulsionar o acesso à informação e ao conhecimento; Facilitar a obtenção de informações disponíveis na Internet.

$\mathrm{Na}$ segunda, os objetivos referem-se ao controle e organização da informação, que contribuem no controle bibliográfico e na adoção e no desenvolvimento de instrumentos de organização da informação e consistem em: Propiciar o acesso aos registros bibliográficos; Criar metodologias e instrumentos para organização da informação; Ser instrumento de controle bibliográfico; Produzir catálogos coletivos; Facilitar e desenvolver os processos e fluxos da organização da informação; Intercambiar registros bibliográficos; Desenvolver mecanismos de organização das informações disponíveis na Internet. 
Em uma dimensão baseada na disseminação (terceira) os objetivos estão alicerçados na difusão da informação e do conhecimento e estabelecem os meios e recursos para a propagação da informação, e constituem-se em: Desenvolver serviços e produtos de informação a comunidades específicas; Prestar subsídios informacionais às ações científicas, tecnológicas e industriais; Agregar valor às informações disponíveis na Internet.

E na última dimensão, os objetivos arrolam a gestão das redes, o que significa direcionar o processo de organização e funcionamento das próprias redes, mediante planejamento e organização de ações orientadas à cooperação e ao crescimento individual e coletivo, e consistem em: Compartilhar recursos e serviços de informação; Implementar o uso de novas tecnologias e o desenvolvimento de ações e instrumentos que as utilizem; Automatizar coleções bibliográficas; Diminuir custos, otimizando recursos; Agilizar processos, capacitando pessoas e ganhando tempo; Impulsionar o desenvolvimento da informação em C\&T; Criar mecanismos para o desenvolvimento de produtos e serviços personalizados.

Pelos objetivos podemos observar que houve um avanço nas atividades das redes, que ultimamente contemplam espaços diferenciados - o real e o virtual -, e recursos e serviços coadunados às tecno- logias da informação, mas mesmo assim essas iniciativas precisam de maior articulação e desenvolvimento para que de fato possamos atingir todas as áreas do conhecimento. É necessário maior empenho e capacitação profissional para empreendermos ações que possam integrar mais e gerar maiores benefícios.

A participação brasileira em rede ainda é pequena, a maior parte dos serviços e unidades de informação insere-se em redes tradicionais, que existem há cerca de 15 anos ou mais. Tais redes estão trabaIhando apoiadas em recursos tecnológicos e em pessoal especializado e mantendo suas funções. Mas poucas redes estão desenvolvendo ações alternativas e inovadoras, em relação às mudanças que estamos presenciando na sociedade da informação e do conhecimento em que estamos inseridos.

A partir da Internet, as redes passaram a utilizar seus recursos, modernizando-se e aproximando seus membros. Podemos identificar as redes que a Internet modificou, redes essas que inovaram seus processos, adotando, por exemplo, formatos $\mathrm{XML}$ e produzindo bibliotecas virtuais. E identificamos também redes que a Internet possibilitou. São as redes que a partir dos portais puderam organizar e fornecer seus produtos e serviços e as que a partir das informações disponíveis na própria Internet estabeleceram e criaram recursos 
informacionais. Das redes identificadas

pelo levantamento que deu origem a este trabalho, estamos considerando redes inovadoras as que a Internet possibilitou. râmetros, identificamos características presentes nas redes tradicionais e nas redes que estão surgindo (Quadro 3), principalmente no âmbito da Internet.

Apesar do pequeno número de redes inovadoras, que trabalham com novos pa-

\begin{tabular}{|l|l|}
\hline \multicolumn{1}{|c|}{ Funções Tradicionais } & \multicolumn{1}{c|}{ Funções Inovadoras } \\
\hline Mudanças lentas e previsíveis & Mudanças rápidas, dinâmicas e incertas \\
\hline Processos complexos & Processos simples \\
\hline Foco em documentos & Foco na informação \\
\hline $\begin{array}{l}\text { Seus serviços e produtos fornecem indica- } \\
\text { ções bibliográficas }\end{array}$ & $\begin{array}{l}\text { Seus serviços e produtos fornecem a informa- } \\
\text { ção propriamente }\end{array}$ \\
\hline Eqüidade & Possibilita a personalização \\
\hline
\end{tabular}

Quadro 3 - Características das redes que mantêm funções tradicionais e das que mantêm funções inovadoras

$A$ inserção em rede é essencial para que os serviços e as unidades de informação cumpram seus propósitos e consigam trabalhar com informação, atendendo as necessidades e expectativas de sua clientela. O compartilhamento da informação e a criação e implementação de parcerias torna-se cada vez mais a base para o fortalecimento de ações que possibilitam efetivamente o acesso à informação nas diferentes áreas do conhecimento.

\section{REFERÊNCIAS}

BARBOSA, Alice Príncipe. Novos rumos da catalogação. Rio de Janeiro: BNG/Brasilart, 1978.

ELKINGTON, Nancy E.; MASSIE, Dennis. The changing nature of international resource sharing: risks and benefits of collaboration. Interlending \& Document Supply, v.27, n.4, p.148-153, 1999.
GUINCHAT, Claire; MENOU, Michel. Introdução geral às ciências e técnicas da informação e documentação. 2.ed. Brasília: IBICT, 1994.

KATZ, William A. Introduction to reference work. 7.ed. New York: The MacGraw-Hill, 1997. 2v.

LOZANO, Marta. Redes de información: conceptos e historia. In: SIMPOSIO ELECTRÓNICO REDES DE BIBLIOTECAS: oportunidad para el cambio. Buenos Aires, 6 a 28 de maio de 2004.

McCALLUM, Sally H. Metadata, protocol, and identifier activities: Library of Congress IFLA/CDNL Alliance for Bibliographic Standards Report. In: WORLD LIBRARY AND INFORMATION CONGRESS: IFLA General Conference and Council, 70. Proceedings... Buenos Aires, 22-27 Aug. 2004. Disponível em:

<http://www.ifla.org/IV/ifla70/papers/024eMcCallum.pdf>. Acesso em: 16 ago. 2004.

MCGARRY, Kevin. O contexto dinâmico da informação: uma análise introdutória. Brasília: Briquet de Lemos/Livros, 1999. 
PACKER, Abel Laerte. O papel da rede brasileira de Informação em Ciências da Saúde na construção da biblioteca virtual em Saúde. In: REUNIÃO DA REDE BRASILEIRA DE INFORMAÇÃO EM ClÊNCIAS DA SAÚDE, 8. Florianópolis, 25 de abril de 2000. Disponível em: $<$ http://www.bireme.br/bvs/snbu/snbu_doc. $\mathrm{htm}>$. Acesso em: 15 jun. 2004.

RODRIGUES, Ricardo. Programa de comutação bibliográfica COMUT. In: WORLD LIBRARY AND INFORMATION CONGRESS: IFLA General Conference and Council, 70. Proceedings... Buenos Aires, 22-27 Aug. 2004. Disponível em: <http://www.ifla.org/IV/ifla70/papers/150sRodrigues.pdf>. Acesso em: 16 ago. 2004.

ROWLEY, Jennifer. Informática para bibliotecas. Brasília: Briquet Lemos/Livros, 1994. Cap. 15, p.285-302.

SILVA, Edna Lúcia da. Compartilhamento de recursos e o papel das redes de informação. Revista de Biblioteconomia de Brasília, Brasília,v.14, n.2, p.209-225, jul./dez. 1986.

VIEIRA, Anna da Soledade. Redes de ICT e a participação brasileira. Brasília: IBICT, 1994.

WEISZ, Joel; ROCO, Mihail C. Rede de pesquisa em educação em engenharia nas Américas. Rio de Janeiro: FINEP, 1996.

ZAHER, Célia Ribeiro. Consorcio electrónico de bibliotecas: um plan de cooperación bibliográfica. In: WORLD LIBRARY AND INFORMATION CONGRESS: IFLA General Conference and Council, 70. Proceedings... Buenos Aires, 22-27 Aug. 2004. Disponível em: <http://www.ifla.org/IV/ifla70/papers/010s_t rans-Ribeiro-Zaher.pdf>. Acesso em: 16 ago. 2004.
Maria Inês Tomaél

Professora do Departamento de Ciência da Informação da Universidade Estadual de Londrina. Mestre em Educação (UEL). Doutora em Ciência da Informação (Escola de Ciência da Informação - UFMG) mitomael@uel.br

\section{Title}

Information Networks: The Contact Point of Information Services and Units in Brazil

\begin{abstract}
The information services and units, organized in networks, perform a vital role in the information management processes, since the acquisition, organization, and dissemination, until the information attainment by the final user. With the objective of raising the participation of the information services and units in networks in Brazil, it was carried out an investigation, in which were consulted, especially, the web sites of the most important Brazilian university libraries, which resulted in a mapping of the current networks in Brazil. It was identified the Brazilian participation in 41 networks, which were categorized according to their function. The category with the largest number of networks is the specialized information; however, it was also found networks of information matching, networks of information services, networks of information processing, and networks of digital information. The participation in a network increases the institutional and informational assets, and, in the contemporary society, it has become, more and more, the foundation for actions strengthening.
\end{abstract}

\section{Keywords}

Information Networks, Cooperative Services, Information Services and Units 


\section{Título}

Redes de Información: el Punto de Contacto de los Servicios y Unidades de Información en Brasil

\section{Resumen}

Los servicios y las unidades de información, organizados en red, ejercen una función esencial en los procesos de la gestión de la información, desde la aquisición, organización, diseminación hasta la obtención de la información por el usuario final. Con el objetivo de levantar la participación de los servicios y unidades de información en redes, en Brasil, procedemos a un levantamiento, en que consultamos, especialmente, los sites de las principales bibliotecas universitárias que resultó en un mapeamento de las redes presentes en Brasil. Identificamos la participación brasileña en 41 redes, que fueron categorizadas de acuerdo con su función. La categoría que detiene el mayor número de redes es la de información especializada, mientras encontramos, también, redes de compatibilización de la información, redes de servicios de información, redes de procesamiento de información y redes de información digital. La participación en red aumenta los activos institucionales e informacionales y en la sociedad contemporánea son, cada vez más, la base para el fortalecimiento de acciones.

\section{Palabras Clave}

Redes de Información, Servicios Coperativos, Servicios y Unidades de Información 